\title{
Insight and treatment attitude in schizophrenia: comparison of patients on depot and atypical antipsychotics
}

\author{
AIMS AND METHOD \\ To establish if participants with \\ schizophrenia receiving depot anti- \\ psychotics had less insight than \\ similar participants receiving oral \\ atypical antipsychotics. We assessed \\ the difference between these two \\ groups.
}

\author{
RESULTS \\ Participants on oral antipsychotics \\ had greater insight than those on \\ depot antipsychotics (ITAQ, $P=0.01$ ). \\ In the multiple regression analysis, \\ only receiving depot antipsychotics \\ contributed significantly to \\ explaining variance in insight \\ (adjusted $R^{2}=0.135, F=8.99$, \\ $P=0.004)$.
}

\begin{abstract}
CLINICAL IMPLICATIONS
Depot antipsychotics seem to be prescribed to a subgroup of people with schizophrenia who are likely to be less adherent because of lower levels of insight. These individuals are on significantly higher doses of antipsychotic medication. Clinicians should review their patients on depot antipsychotics at regular intervals.
\end{abstract}

The concept of insight as it applies to psychiatry is a complex phenomenon. David (1990) proposed that insight consists of three overlapping dimensions: the recognition that one has a mental illness, adherence with treatment and the ability to re-label unusual mental events (delusions and hallucinations) as pathological.

Lack of insight is a common symptom of the acute phase of schizophrenia, being described in $97 \%$ of acute cases in the World Health Organization International Pilot Study of Schizophrenia (World Health Organization,

1973). Lack of insight often responds to treatment, but it also persists in a substantial proportion of people with schizophrenia (Cuesta et al, 2000), and tends to be associated with non-adherence (Mcevoy et al, 1989a; Sanz et al, 1998).

Non-adherence itself is associated with relapse, rehospitalisation (Haywood et al, 1995; Fenton et al, 1997) and social breakdown, which result in substantial hardships and cost to patients, their families, and society as a whole. Depot antipsychotic medication is commonly prescribed in cases of poor adherence, particularly when covert non-adherence is suspected (Valenstein et al, 2001). Administration of a depot ensures either that a patient receives adequate levels of medication or that their failure to receive medication is detected early (through refusal or failure to attend for depot administration). However, long-term treatment with depot antipsychotics has disadvantages. In particular, it is associated with an increased risk of extrapyramidal sideeffects, tardive dyskinesia, weight gain and depression (Cookson, 1991). Depot medication involves painful injections, may require attendance at depot or primary care clinics, could make patients feel less in control of their illness, and dose adjustments to reach optimal dose can be time consuming.

Over the past decade, the introduction of atypical antipsychotics has been accompanied by a reduction in the use of depot antipsychotics (Patel \& David, 2005). A substantial number of patients have switched from depot antipsychotics to oral atypicals with apparently beneficial results. For example, an observational study by Desai et al (1999) reported that switching from depot antipsychotics to risperidone tablets resulted in significant improvement of positive and negative symptoms, level of functioning, parkinsonism and dyskinesia.

However, a substantial number of patients remain on depot antipsychotic medication long term. This raises the question of whether this is due to inertia, or reflects a rational clinical decision based on the benefits of reducing covert non-adherence in a subgroup of patients with poor insight. To answer this question, we tested the hypothesis that patients with schizophrenia on depot medication would have lower levels of insight than similar patients receiving oral antipsychotics. We also aimed to control for potential confounding variables such as symptom severity, side-effects and duration of illness.

\section{Method}

A cross-sectional assessment was undertaken of stable community patients with schizophrenia at two sites in the north west of England (Preston and Blackburn), both served by Lancashire Care NHS Trust. The local research ethics committee approved the study.

The inclusion criteria were that participants were aged 18-65 years, had an ICD-10 (World Health Organization, 1992) diagnosis of schizophrenia, had been stable in the community for the previous 6 months, and were currently prescribed either depot or oral antipsychotic medication. Patients who were prescribed clozapine were excluded, as they were considered to represent a subgroup that would be pre-selected for treatment resistance, as were patients who were taking both oral and depot medication. Written informed 
consent was obtained from all participants. A pre-study power calculation specified that 26 participants per group were required for an $80 \%$ power to show a

original papers statistically significant difference between the two groups at the $5 \%$ significance level, using a two-sided t-test.

Basic demographic, clinical and social characteristics were collected using a structured questionnaire. Insight and attitude to treatment was assessed using the Insight and Treatment Attitude Questionnaire (ITAQ) (Mcevoy et al, 1989b). The ITAQ is a semi-structured interview of 11 items that measures awareness of illness (first 5 items) and attitude to medication/hospitalisation and follow-up evaluation (6 items). Scores range from 0 (no insight) to 22 (full insight).

The Brief Psychiatric Rating Scale (BPRS; Overall \& Gorham, 1962) was used to assess psychopathology. The BPRS contains 16 items that measure negative symptoms (2 items), positive symptoms (5 items) and general psychopathology (9 items).

Akathisia was assessed using the Barnes Akathisia rating scale (BARS; Barnes, 1989). This scale is observer rated and includes items for the objective and subjective aspects of akathisia as well as a global rating. The Extra Pyramidal Side Effects Scale (ESES; Mcevoy et al, 1991) was used to assess bradykinesia, rigidity and tremor. Tardive dyskinesia was assessed using the Abnormal Involuntary Movements Scale (AIMS; Guy \& Ban, 1979).

The dosage of each antipsychotic was converted to its chlorpromazine equivalents (Lehman \& Steinwachs, 1998; Woods, 2003).

The Statistical Package for Social Science (SPSS) for Windows, Version 10 was used for the analysis. Univariate tests were used to assess differences between the depot antipsychotic group and the oral antipsychotic group. For all tests, the level of significance $(P)$ was set at the conventional level of 0.05 (two-sided).

Multiple regression was used to analyse the relative contribution of symptom severity (BPRS score), sideeffects (BARS, ESES and AIMS scores), duration of illness

Table 1. Characteristics of the depot and oral antipsychotic groups

\begin{tabular}{|c|c|c|c|}
\hline Variable & Depot & Oral & $P$ \\
\hline$n$ & 26 & 26 & \\
\hline Age, years (s.d.) & $44.4(11.87)$ & $42.0(13.65)$ & 0.51 \\
\hline \multicolumn{4}{|l|}{ Gender, $n(\%)$} \\
\hline Male & $21(80.77)$ & $19(73.07)$ & 0.74 \\
\hline Female & $5(19.23)$ & $7(26.92)$ & \\
\hline \multicolumn{4}{|c|}{ Marital status, $n(\%)$} \\
\hline Married & $3(11.54)$ & $7(26.92)$ & 0.35 \\
\hline Divorced & $6(23.08)$ & $4(15.38)$ & \\
\hline Single & $17(65.38)$ & $15(57.69)$ & \\
\hline \multicolumn{4}{|c|}{ Employment, n (\%) } \\
\hline Employed & $1(3.85)$ & $1(3.85)$ & 0.60 \\
\hline Unemployed & 25 (96.15) & $24(92.31)$ & \\
\hline Student & 0 & $1(3.85)$ & \\
\hline \multicolumn{4}{|c|}{ Living situation, $n(\%)$} \\
\hline Alone & $11(42.31)$ & $4(15.38)$ & 0.06 \\
\hline With family & $7(26.92)$ & $14(53.85)$ & \\
\hline Other & $8(30.77)$ & $8(30.77)$ & \\
\hline
\end{tabular}

and group membership (depot or oral) to insight (ITAQ scores).

\section{Results}

We recruited 52 participants, of which 26 were receiving depot medication and 26 were on oral medication. The demographic characteristics of the participants are summarised in Table 1. The mean age of participants was 44.4 years (s.d. $=11.9)$ in the depot group and 42.0 years (s.d.=13.7) in the oral group. In both groups the majority of the participants were male (depot $80.7 \%$; oral $73.1 \%$ ) and single (depot $65.4 \%$; oral $57.7 \%$ ). More participants from the depot group were living alone (depot $42.3 \%$; oral 15.4\%) and the majority of participants were unemployed (depot 95.2\%; oral 92.3\%). No differences reached statistical significance.

In the oral antipsychotic group all the participants were on atypical antipsychotics. In the depot group 23 participants were on typical depot medication and 3 were on risperidone long-acting injection. The mean chlorpromazine equivalent dose of antipsychotic was much higher in the depot group than the oral antipsychotic group (1388.7, s.d. $=1870.7$ v. 391.0, s.d. $=205.5 ; z=-3.16$, $P=0.002$ ).

Illness variables for the two groups are summarised in Table 2. The mean age at onset of psychotic symptoms for the whole sample was 26.1 years (s.d. $=8.8$ ) and the mean duration of illness was 17.2 years (s.d. $=9.7$ ). The mean duration of illness was significantly longer for the depot group than the oral group (20.2, s.d. $=9.5$ v. 14.1, s.d. $=9.1 ; P=0.02,95 \% \mathrm{Cl} 0.9$ to 11.3). More patients from the depot group had a history of compulsory admission under the Mental Health Act $(76.9 \%$ v. 53.9\%, $P=0.07)$ compared with the oral group.

The mean BPRS score of the total sample was 28.0 (s.d.=7.9). The depot group had a slightly better mean

Table 2. Illness variables and side-effects scores between the depot $(n=26)$ and oral $(n=26)$ antipsychotic groups

\begin{tabular}{lccc}
\hline & Depot & Oral & $P$ \\
\hline $\begin{array}{l}\text { Age of onset, } \\
\text { mean (s.d.) }\end{array}$ & $24.3(6.64)$ & $28.0(10.34)$ & 0.22 \\
Duration of illness, & & & \\
mean (s.d.) & $20.2(9.50)$ & $14.1(9.12)$ & $0.02^{*}$ \\
Involuntary admission, & & & \\
n (\%) & & & \\
Yes & $20(76.92)$ & $14(53.85)$ & 0.07 \\
$\quad$ No & $6(23.08)$ & $12(46.15)$ & \\
Side-effect scores & & & \\
BPRS, mean (s.d.) & $27.0(7.37)$ & $29.0(8.51)$ & 0.36 \\
BARS, mean (s.d.) & $2.5(2.56)$ & $1.5(2.12)$ & 0.15 \\
ESES, mean (s.d.) & $1.0(1.51)$ & $0.9(1.35)$ & 0.79 \\
AIMS, mean (s.d.) & $2.4(2.26)$ & $1.3(2.8)$ & $0.004^{*}$ \\
\hline
\end{tabular}

AIMS, Abnormal Involuntary Movements Scale; BARS, Barnes Akathisia Rating Scale; BPRS, Brief Psychiatric Rating Scale; ESES, Extra Pyramidal Side Effects Scale.

*These values are statistically significant. 
Table 3. ITAQ scores in the depot $(n=26)$ and oral $(n=26)$

antipsychotic group

\begin{tabular}{|lccc}
\hline & Depot & Oral & $P$ \\
\hline $\begin{array}{l}\text { ITAQ scores, mean (s.d.) } \\
\text { Total score }\end{array}$ & $16.3(3.99)$ & $19.0(2.25)$ & $0.01^{*}$ \\
$\begin{array}{l}\text { Awareness of illness } \\
\text { (items 1-5) }\end{array}$ & $6.9(2.04)$ & $8.0(1.32)$ & $0.03^{*}$ \\
$\begin{array}{l}\text { Attitude to treatment } \\
\text { (items 7-11) }\end{array}$ & $9.4(2.35)$ & $10.9(1.37)$ & $0.01^{*}$ \\
\hline
\end{tabular}

ITAQ, Insight and Treatment Attitude Questionnaire.

*These values are statistically significant.

BPRS score than the oral group but this difference was not statistically significant $(27.0$, s.d. $=7.4$ v. 29.0, s.d. $=8.5 ; P=0.36,95 \% \mathrm{Cl}-6.5$ to 2.4$)$.

Overall the level of insight was fairly high, with the total sample having a mean ITAQ score of 17.6 (range 1022 , s.d.=3.5). However, the patients on oral medication had a statistically significantly higher mean score than those on depot $(16.3$, s.d. $=4.0$ v. 19.0, s.d. $=2.3 ; z=-2.4$, $P=0.01$ ). A sub-score analysis of the ITAQ showed that the oral medication group scored significantly higher than the depot group on both awareness of illness and attitude to treatment (Table 3).

Overall the sample had relatively low levels of sideeffects (Table 2). There was no significant difference between the two groups other than for the tardive dyskinesia (AIMS score)

Total scores for BPRS, AIMS, ESES and BARS, duration of illness and group were entered as independent variables in a stepwise multiple regression analysis, with ITAQ score (as the dependent variable). Only group (depot or oral) contributed significantly to the regression model (adjusted $R^{2}=0.135, F=8.99, P=0.004$ ).

\section{Discussion}

The participants in this study had relatively high levels of insight into their illness. The study sample consisted of stable out-patients. This finding is consistent with previous reports of higher insight among out-patients with schizophrenia (Garavan et al, 1998; Williams \& Collins, 2002).

The findings of the study confirmed our hypothesis that participants receiving depot antipsychotics would have significantly less insight than those receiving oral antipsychotics. This result was supported by our additional finding that participants on depots were also more likely to have been admitted to hospital under the Mental Health Act, suggesting that they were less adherent with medication than the group receiving oral antipsychotics. This suggests that psychiatrists were taking adherence and/or insight into account when deciding to start and maintain patients on depot medication.

It can be hypothesised that the majority of the participants on oral antipsychotics were started and maintained on oral antipsychotics because of their better

insight and adherence. However, because of the crosssectional nature of the study design, it is not possible to ascertain the insight of the participants when they were started on their medication.

The finding of poorer insight among participants taking depot medication is open to an alternative explanation that the depot medication was less effective than the oral medication at improving insight. However, against this explanation is the fact that the two groups had similar levels of symptomatology. This finding, together with the similar level of side-effects between the two groups, reflects the broader evidence base that depots are as effective and safe as oral antipsychotic medication for the treatment of schizophrenia (Adams et al, 2001).

Insight was shown to be a clinical modulator of long- and short-term adherence with treatment and is a good indicator of prognosis (Buchanan, 1992). The clinical relevance of the findings from this study is that appropriate interventions should be offered to patients on depot medication to improve their insight. This will have a beneficial effect on adherence and long-term prognosis.

We found that the participants on depot antipsychotics were on significantly higher doses of antipsychotic medication compared with those on atypical medication, to the ratio of 1:35. The participants on depot medication were on a mean chlorpromazine equivalent dose of $1388.7 \mathrm{mg}$; well above the recommended range of 300-1000 mg chlorpromazine equivalent. Clinicians should review the patients on depot antipsychotics at regular intervals and review the dose of their depot medication.

It is possible that the findings of the study are only locally applicable to the sites in east Lancashire, as the study is relatively small and restricted to patients from this area. None the less, as far as we are aware, this study provides the best evidence so far that the decision to put patients on depot rather than oral medication is being made on a rational basis. Future studies should address this question in larger cohorts of patients, where changes in adherence are observed over time from the inception of the antipsychotic medication.

\section{Declaration of interest}

None.

\section{Acknowledgements}

We thank Dr Saleem and Maureen Eka Harrison (Queens Park Hospital, Blackburn) for their help in recruiting participants. These findings were presented as a poster at the Royal College of Psychiatrists Annual General Meeting of the Royal College of Psychiatrists, Edinburgh, June 2005.

\section{References}

ADAMS, C. E., FENTON, M. K. P. QUARAISHI, S., et al (2001) Systematic meta-review of depot antipsychotic drugs for people with schizophrenia. British Journal of Psychiatry, 179, 290 299. original papers 
BARNES, T. R. E. (1989) A rating scale for drug induced akathisia. British Journal of Psychiatry, 154, 672-679.

original papers

BUCHANAN, A. (1992) A two-yea prospective study of treatment compliance in patients with schizophrenia. Psychological Medicine, 22, 787-797.

COOKSON, J. C. (1991) Side effects during long-term treatment with depot anti-psychotic medication. Clinical Neuropharmacology, 14 \$24-\$32.

CUESTA, M. J., PERALTA, V. \& ZARZUELA, A. (2000) Re appraising insight in psychosis: multiscale longitudinal study. British Journal of Psychiatry, 177, 233-240.

DAVID, A. (1990) Insight and psychosis. British Journal of Psychiatry, 156, 798 808.

DESAI, N. M., HUQ, Z., MARTIN, S. D., et al(1999) Switching from depot antipsychotics to risperidone: results of a study of chronic schizophrenia. The

SchizophreniaTreatment and

Assessment Group. Advances in

Therapy, 16, 78-88.

FENTON, W. S., BLYLER, C. R. \&

HEINSSEN, R. K. (1997) Determinants of medication compliance in

schizophrenia: empirical and clinica findings. Schizophrenia Bulletin, 23 637-651.

GARAVAN, J., BROWNE, S., GERVIN M., et al (1998) Compliance with neuroleptic medication in outpatients with schizophrenia; Relationship to subjective response to neuroleptics; attitudes to medication and insight. Comprehensive Psychiatry, 39, 215219.

GUY,W. \& BAN, T. A. (1979) The AMDP System. Springer.

HAYWOOD,T.W., KRAVITZ, H. M. GROSSMAN, L. S., et al (1995)

Predicting the 'revolving door' phenomenon among patients with schizophrenic, schizoaffective, and affective disorders. American Journal of Psychiatry, 152, 856-861.

LEHMAN, A. F \& STEINWACHS, D. M. (1998) Translating Research Into Practice: The Schizophrenia Patient Outcomes ResearchTeam (PORT)

Treatment Recommendations. Schizophrenia Bulletin, 24, 1-10. MCEVOY, J. P., APPLEBAUM, P. S., APPERSON, L. J., et al (1989a) Why must some schizophrenic patients be involuntarily committed? The role of insight. Comprehensive Psychiatry, $\mathbf{3 0}$ 13-17

MCEVOY, J. P., APPERSON, L. G. APPLEBAUM, P. S., et al (1989b) Insight in schizophrenia: Its relation to acute psychopathology. Journal of Nervous and Mental Disease, 177, 43-47.

MCEVOY, J. P., HOGART, G. E. \& STEINGARD, S. (1991) Optimal dose of neuroleptic in acute schizophrenia. Archives of General Psychiatry, $\mathbf{4 8}$ 739-745.

OVERALL, J. E. \& GORHAM, D. R. (1962) The Brief Psychiatric Rating Scale. Psychological Report, 10,799-812.

PATEL, M. X. \& DAVID, A. S. (2005) Why aren't depot antipsychotics prescribed more often and what can be done about it? Advances in Psychiatric Treatment, 11, 203-213.

SANZ, M., CONSTABLE, G., LOPEZ IBOR, I., et al (1998) A comparative study of insight scales and their relationship to psychopathological and

clinical variables. Psychological Medicine, 28, 437-446.

VALENSTEIN, M., COPELAND, L. A OWEN, R., et al (2001) Adherence assessments and the use of depot antipsychotics in patients with schizophrenia. Journal of Clinical Psychiatry, 62, 545-551.

WILLIAMS, C. C. \& COLLINS, A. (2002) Factors associated with insight among outpatients with serious mental illness. Psychiatric Services, 53, 96-98.

WOODS, S.W. (2003) Chlorpromazine equivalent doses for the newer atypical antipsychotics. Journal of Clinical Psychiatry, 64, 663-667.

WORLD HEALTH ORGANIZATION (1973) Report of the International Pilot of Schizophrenia (Vol. 1).WHO

WORLD HEALTH ORGANIZATION (1992) The ICD-10 Classification of Menta and Behavioural Disorders. Clinical Descriptions and Diagnostic Guidelines. WHO.

*P. N. Mahadun Bolton Salford and Trafford Mental Health NHS Trust, Crisis resolution HomeTreatment Team, Chapel Road, Sale, Manchester M337EG, email: prem.mahadun@trafford.nhs.uk, M. Marshall School of Psychiatry and Behavioural Sciences, University of Manchester, Preston

\section{Capacity assessments on medical in-patients referred to social workers for care home placement}

\section{AIMS AND METHODS}

To investigate the feasibility of a clinical algorithm to assess capacity and examine the relationship between its results and the assess ments of capacity by others involved in the decision of a patient to permanently enter a care home from a medical ward.

\author{
RESULTS \\ A total of 23 patients out of 38 \\ $(60.5 \%, 95 \% \mathrm{Cl} 44-77)$ had some \\ mention of capacity in any type of \\ record (medical, social work or \\ nursing). At formal assessment \\ $47 \%$ of older patients lacked \\ capacity.
}

\begin{abstract}
CLINICAL IMPLICATIONS
The absence of any recorded assessment in at least a third of patients is worrying, given the importance of the decision to the patients' lives and their financial status. It is to be hoped that the implementation of the Mental Capacity Act (2005) will rectify this situation.
\end{abstract}

The definition of capacity given in the Mental Capacity Act for England and Wales (2005) is that, at the time a decision needs to be made, a person is able to understand the information relevant to the decision, retain that information, use or weigh that information as part of the process of making the decision, and communicate his decision (whether by talking, using sign language or any other means)

Capacity is specific to the task in hand. In ambiguous circumstances the risks associated with the decision must be proportionate to the degree of certainty of the person's capacity, a 'sliding scale' of capacity (Stone, 1994). The assessment of capacity is subjective and can be complex.
Doctors are often asked or take upon themselves to evaluate the ability of older adults to continue living alone in the community; their capacity to make this decision can be more difficult to assess than that for other medical dilemmas. A large number of requests for capacity assessments of medical in-patients are seen in liaison psychiatric services for older people (Mujic et al, 2004): the role of the psychiatrist should be to assess the degree of impairment that may affect capacity. The assessment of the capacity of older people to consent to permanently enter a care home is a major issue. Pressure on beds may preclude both older people and their carers from exercising a genuinely informed choice (Lundh et al, 2000). It is usually a family member who takes the lead in 